\title{
Magnetic Multilayers as a Way to Increase the Magnetic Field Responsiveness of Magnetocaloric Materials
}

\author{
R. Caballero-Flores ${ }^{1}$, V. Franco ${ }^{1, *}$, A. Conde ${ }^{1}$, L. F. Kiss ${ }^{2}$, L. Péter ${ }^{2}$, and I. Bakonyi ${ }^{2}$ \\ ${ }^{1}$ Dpto. Física de la Materia Condensada, ICMSE-CSIC, Universidad de Sevilla, P.0. Box 1065, 41080 Sevilla, Spain \\ ${ }^{2}$ Wigner Research Centre for Physics, Hungarian Academy of Sciences, \\ H-1525 Budapest, P.O. Box 49, Hungary
}

\section{INTRODUCTION}

Solid-state magnetic refrigeration at room temperature based on the magnetocaloric effect (MCE) is a field of active research in recent years. ${ }^{1-3}$ Its energy-efficient and environment-friendly features are the prominent advantages over conventional gas compression-expansion refrigeration technology that is used nowadays. ${ }^{4}$

The MCE is the reversible temperature change $\left(\Delta T_{\mathrm{ad}}\right)$ of a magnetic material due to the application of an external magnetic field change $(\Delta H)$ under adiabatic conditions. Alternatively, the MCE can be characterized as the reversible magnetic entropy change $\left(\Delta S_{M}\right)$ experimented by the magnetic material due to the application of an external magnetic field $H$ at temperature $T$ under isothermal conditions:

$$
\Delta S_{M}(T, \Delta H)=\left.\int_{H_{0}}^{H_{f}} \mu_{0} \frac{\partial M(T, H)}{\partial T}\right|_{H} d H
$$

where $\Delta H=H_{f}-H_{0}, \mu_{0}$ is the magnetic permeability of vacuum, and $M(T, H)$ is the magnetization of the material. In this work, it is considered that $\mu_{0} H_{0}=0 \mathrm{~T}$.

Along with the $\Delta S_{M}$ values, magnetic refrigerants are also characterized by the refrigerant capacity ${ }^{5}(R C)$ defined as the heat transferred per unit volume of the refrigerant material from the hot (at temperature $T_{\text {hot }}$ )

\footnotetext{
${ }^{*}$ Author to whom correspondence should be addressed.
}

to the cold (at temperature $T_{\text {cold }}$ ) reservoirs in the used thermodynamic cycle, which is associated with the width of the $\Delta S_{M}(T)$ curve

$$
R C(\delta T, H)=\int_{T_{\text {cold }}}^{T_{\text {hot }}} \Delta S_{M}(T, H) d T
$$

where $\delta T=T_{\text {hot }}-T_{\text {cold }}$. In the literature, it is usual to determine the value of $\delta T$ according to the temperature values corresponding to the full width at half maximum of the $\Delta S_{M}(T)$ curve $\left(\delta T_{\mathrm{FWHM}}\right)$, yielding in this case the definition of the refrigerant capacity denoted as $R C_{\mathrm{AREA}}$.

In order to make the magnetic refrigeration technology feasible for the domestic market, there is the need to find materials with large $\Delta S_{M}$ and $R C$ values in the environment of the working temperature of the refrigerator, and that these magnitudes are achieved with the application of a moderate magnetic field.

According to the experimental results, it can be assumed that the field dependence of $\Delta S_{M}$ and $R C$ follow power laws of the field ${ }^{6,7}$

$$
\begin{gathered}
\Delta S_{M}(T, H)=a(T) H^{n(T, H)} \\
R C_{\mathrm{AREA}}(H)=b H^{\xi}
\end{gathered}
$$

where the exponent $n$, in general, depends on temperature and field. For single-phase materials, the asymptotic values of $n$ are 1 and 2 when the values of temperature are quite 
Caballero-Flores et al. Magnetic Multilayers as a Way to Increase the Magnetic Field Responsiveness of Magnetocaloric Materials

far below and above the Curie temperature $\left(T_{\mathrm{C}}\right)$, respectively. Also for single-phase materials, the minimum value of this exponent at the Curie temperature, $n\left(T=T_{\mathrm{C}}\right)$, is field independent and can be expressed in terms of the critical exponents as $n=1-(1-\beta) /(\beta+\gamma)$. It has been observed experimentally that for typical amorphous alloys the exponent $n\left(T=T_{\mathrm{C}}\right)$ takes a value of the order of 0.7 ( $2 / 3$ for the mean field theory). On the other hand, the exponent $\xi$ that controls the field dependence of the refrigerant capacity $R C_{\mathrm{AREA}}$ is related to the critical exponents as ${ }^{7} \xi=1+\beta /(\beta+\gamma)$ (taking the value $4 / 3$ for the mean field theory). Note that the exponents $n$ and $\xi$ are related via the expression $\xi=n+1 /(\beta+\gamma)$. For multiphase materials, however, it has been shown that they exhibit higher values of the exponent $n$ than single-phase materials in the temperature range between the Curie temperatures of the constituent phases. ${ }^{8}$

The study of MCE using multilayers/superlattices has been recently presented as an alternative method to obtain tunable and improved magnetocaloric materials ${ }^{9}$ with respect to the corresponding polycrystalline or amorphous systems, especially when they present second-order phase transitions. ${ }^{10}$ The goal of the present work is to study the field dependence of $\Delta S_{M}$ and $R C$ in multiphase materials with a multilayer nanostructure, in order to find materials with values of the exponent $n$ higher than 0.7 in a wide temperature span where the MCE is maximum, i.e., in the environment of their transition temperatures. This could be used to design magnetic refrigerant materials with enhanced field responsiveness for moderate magnetic fields.

\section{EXPERIMENTAL DETAILS}

In this work, three $\mathrm{Ni}-\mathrm{Cu}$ based multilayer samples, with phases whose nominal Curie temperatures vary between 192 and $449 \mathrm{~K},{ }^{11}$ have been prepared by a electrodeposition technique using different repetition schemes: sample G1 ( $\square)$ : three periods of nine layers in which the current density and the deposition times per layer have been varied; sample G2 (•): six periods of nine layers with the same current density and deposition times per layer than those for G1; and sample G3 ( $\mathbf{\Delta})$ : three periods of nine layers with the same current density and double deposition times than those for G1 and G2.

Electrodeposition was performed at $50{ }^{\circ} \mathrm{C}$ under current control by using an Elektroflex 453 power source. An acetate-stabilized citrate bath was used with the following composition: nickel sulfate (from $\mathrm{NiSO}_{4}$. $\left.6 \mathrm{H}_{2} \mathrm{O}\right) 0.3 \mathrm{~mol} /$ liter, nickel acetate (from $\mathrm{Ni}\left(\mathrm{CH}_{3} \mathrm{COO}\right)_{2}$. $4 \mathrm{H}_{2} \mathrm{O}$ ) $0.05 \mathrm{~mol} / \mathrm{liter}$, copper sulfate (from $\mathrm{CuSO}_{4} \cdot 5 \mathrm{H}_{2} \mathrm{O}$ ) $0.025 \mathrm{~mol} / \mathrm{liter}$, sodium citrate (from $\mathrm{Na}_{3} \mathrm{C}_{6} \mathrm{H}_{5} \mathrm{O}_{7} \cdot 2 \mathrm{H}_{2} \mathrm{O}$ ) $0.25 \mathrm{~mol} / \mathrm{liter}$, and sodium chloride $(\mathrm{NaCl}) 0.03 \mathrm{~mol} / \mathrm{liter}$. This bath was similar to those used for the preparation of $\mathrm{Ni}-\mathrm{Cu}$ alloys ${ }^{11,12}$ and $\mathrm{NiCu} / \mathrm{Cu}$ multilayers. ${ }^{13-15}$ The application of $\mathrm{Ni}$ acetate made it unnecessary to adjust the solution $\mathrm{pH}^{16}$ The concentration of the bath components was optimized to obtain a slowly increasing $\mathrm{Ni}$ content between 3 and $25 \mathrm{~mA} \mathrm{~cm}^{-2}$ current density. In this current density range, the $\mathrm{Ni}$ content of the d.c. deposits changes between 72 and 82 at.\%, which corresponds to the Curie temperature range of 192 to $449 \mathrm{~K}$ for $\mathrm{Ni}-\mathrm{Cu}$ alloys. Due to the 2-3 at.\% Co content of the resulting deposit, the Curie temperatures were systematically higher than those of the $\mathrm{Ni}-\mathrm{Cu}$ alloys with identical $\mathrm{Cu}$ content.

The field and temperature dependence of $M(T, H)$ of the typically $3 \mathrm{~mm}$ wide, $3 \mathrm{~mm}$ long and $\sim 2-5 \mu \mathrm{m}$ thick ribbon-shaped samples has been measured (up to a value of magnetic field $\mu_{0} H_{f}=1.5 \mathrm{~T}$ applied in the plane of the ribbons and in $10 \mathrm{mT}$ increments, and from $323 \mathrm{~K}$ to $773 \mathrm{~K}$ in $10 \mathrm{~K}$ increments) in a vibrating sample magnetometer.

\section{RESULTS AND DISCUSSION}

The temperature evolution of the extremal values of the derivative with respect to temperature at low field of the experimental specific magnetization data $\sigma(T)\left((d \sigma / d T)_{H}\right)$ can be used to determine the distribution of the Curie temperatures $\left(\Delta T_{\mathrm{C}}\right)$ of the existing phases in the samples. Figure 1 indicates that the studied multiphase systems are constituted by different magnetic phases with a broad Curie temperature distribution $\Delta T_{\mathrm{C}} \sim 150 \mathrm{~K}$. Its determination for the samples G2 and G3 has been obtained from the inflection points of the $\sigma(T)$ curves. It can be seen in Figure 1 that the Curie temperature distribution of the phases present in the sample increases as the total deposition time increases, that for the samples G2 and G3 they vary between $\sim 375 \mathrm{~K}$ and $\sim 525 \mathrm{~K}$, and that they can be higher than the nominal Curie temperature of the monolayer with the largest $T_{\mathrm{C}}(449 \mathrm{~K})$.

The magnetic entropy change $\Delta S_{M}$ caused by the variation of the magnetic field has been calculated by numerical integration of the Maxwell relationship (Eq. (1)). Figure 2 shows the temperature dependence of the $\Delta S_{M}(T)$ curves at a maximum magnetic field $\mu_{0} H_{f}=1.5 \mathrm{~T}$ for the three studied samples G1, G2 and G3. The $\Delta S_{M}(T)$ curves have a broad peak, characteristic of multiphase systems, which emerges from the overlapping of several secondorder phase transitions occurring in the broad temperature span $\Delta T_{\mathrm{C}}$. It must be noted that in the samples studied, the $\left|\Delta S_{M}(T)\right|$ curves have maximum values $\left(\Delta S_{M}^{p k}\right)$ at a temperature of $\sim 500 \mathrm{~K}$, and that the table-like shape of the $\Delta S_{M}(T)$ curves becomes more extended as the total deposition time increases, appearing this table-like shape from $\sim 450 \mathrm{~K}$ and $\sim 420 \mathrm{~K}$ for G2 and G3, respectively. It can be also seen in Figure 2 that the full temperature width at half maximum of the peak $\delta T_{\mathrm{FWHM}}$ has a value of $175 \mathrm{~K}$ (G1 and G3) and $225 \mathrm{~K}(\mathrm{G} 2)$. The reason why these nanocomposite materials exhibit broad $\Delta S_{M}(T)$ peaks is 

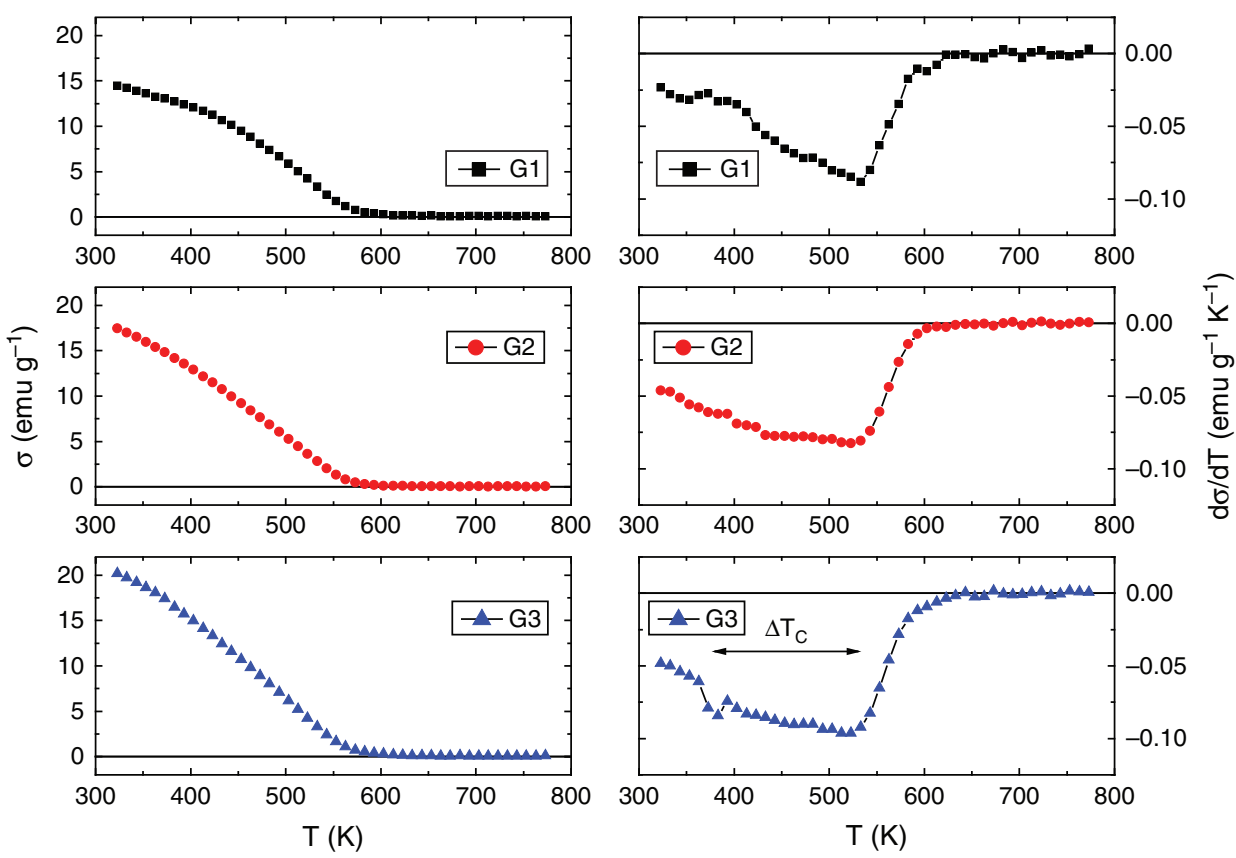

Fig. 1. Temperature dependence of the specific magnetization $\sigma(T)$ and its derivative $(d \sigma / d T)_{H}$ at low field $\left(\mu_{0} H=10^{-3} \mathrm{~T}\right)$ of the studied multiphase samples G1 (upper panels), G2 (central panels) and G3 (lower panels). A Curie temperature distribution of $\Delta T_{\mathrm{C}} \sim 150 \mathrm{~K}$ can be obtained.

the overlapping of the magnetocaloric responses of the existing phases.

The field dependence of the different magnetocaloric responses of the samples has been studied in the whole temperature range defined by the maximum values of the magnetic entropy change $\Delta S_{M}^{p k}$, and this field dependence expressed as $\Delta S_{M}^{p k}(H) \propto H^{n}$ has been fitted according to Eq. (3). Although, in general, $n$ is field dependent for the whole temperature range in multiphase systems, the small field dependence close to the peak lets us to assume a field independent value on $n$ for the fit, otherwise the logarithmic derivative $n(T, H)=d \ln \left|\Delta S_{M}\right| / d \ln H$ must be used.

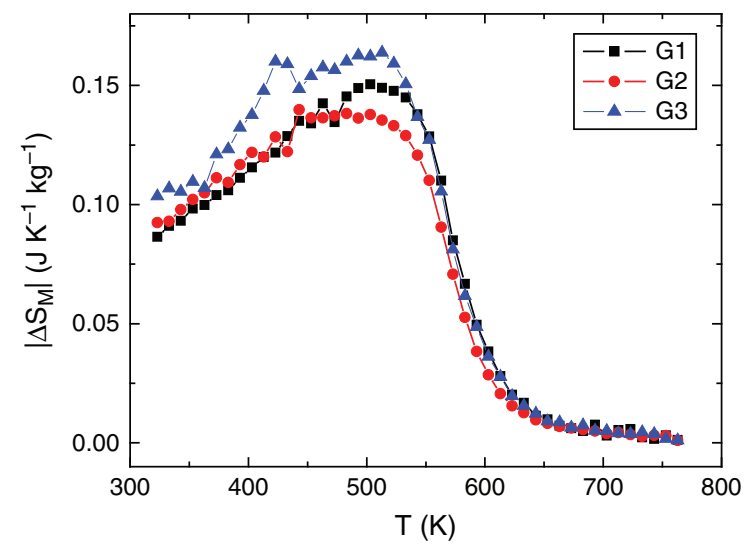

Fig. 2. Temperature dependence of the magnetic entropy change $\Delta S_{M}$ corresponding to an applied field $\mu_{o} H_{f}=1.5 \mathrm{~T}$ of the samples G1 (ם), $\mathrm{G} 2(\bullet)$ and G3 ( $\Delta)$, indicating that all of them exhibit a broad temperature span $\delta T_{\text {FWHM }}$.
The results as presented in Figure 3 indicate that in all the studied cases the field dependence of $\Delta S_{M}$ is nearly linear in the broad temperature span $\Delta T_{\mathrm{C}}$ of the Curie temperatures of the existing phases. The values of the exponent $n$ have been indicated in brackets and this result demonstrates that nanostructured systems are good candidates to control the field dependence of the magnetocaloric response of the materials used in magnetic refrigeration at room temperature.

The field and temperature evolution of the exponent $n$ in the temperature range $\Delta T_{\mathrm{C}}$ for which the

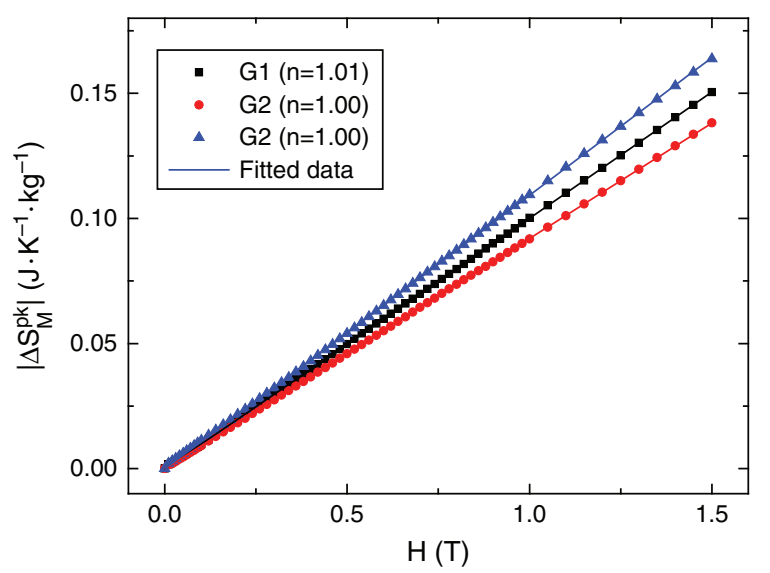

Fig. 3. Field dependence of the maximum entropy change in the studied samples. Symbols correspond to the maximum values at a temperature of $500 \mathrm{~K}(\mathrm{G} 1)$, and any temperature in the ranges from $450 \mathrm{~K}$ to $500 \mathrm{~K}(\mathrm{G} 2)$ or from $420 \mathrm{~K}$ to $500 \mathrm{~K}$ (G3). Solid lines indicate the non-linear fit according to Eq. (3), corresponding to experimental data of the maximum magnetic entropy change values.

J. Nanosci. Nanotechnol. 12, 7432-7436, 2012 
Caballero-Flores et al. Magnetic Multilayers as a Way to Increase the Magnetic Field Responsiveness of Magnetocaloric Materials
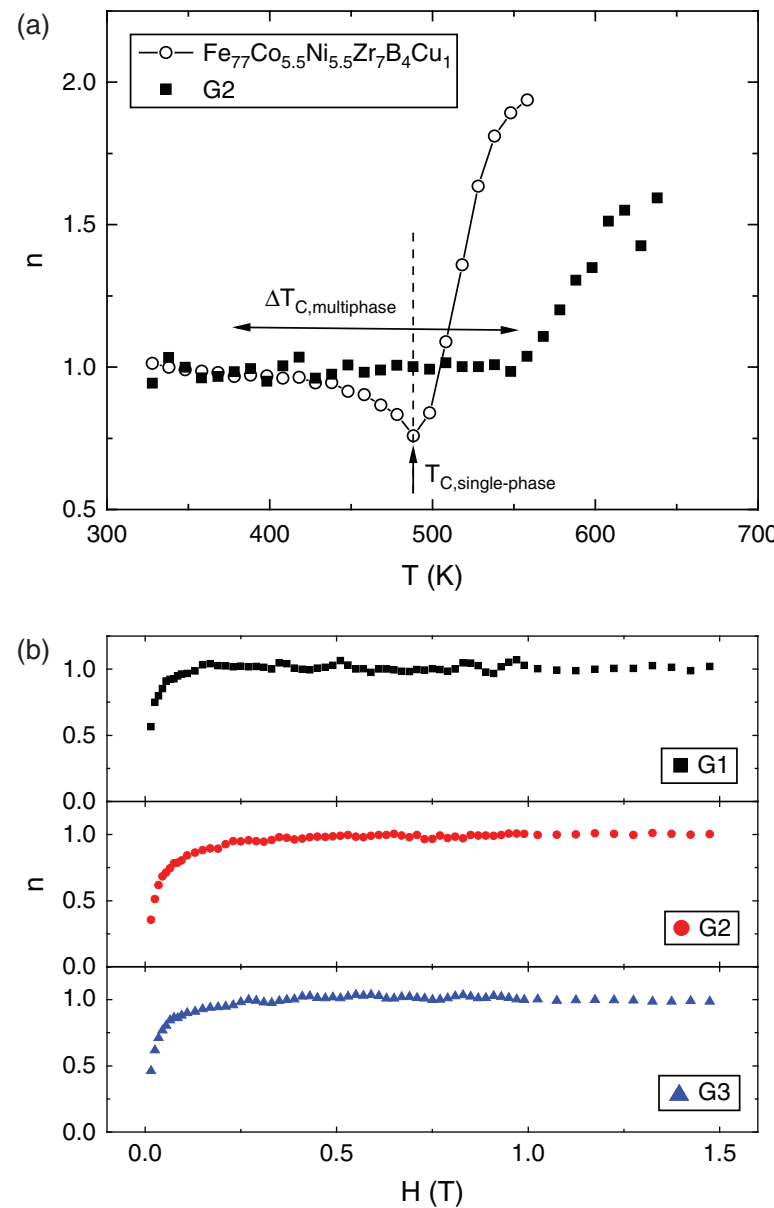

Fig. 4. (a) Temperature dependence of the exponent $n$ at a maximum magnetic field $\mu_{0} H_{f}=1.5 \mathrm{~T}$ for sample G1 (similar behavior for G2 and G3 has been found). (b) Field dependence of the exponent $n$ in the studied samples at the temperatures of the peaks of the $\Delta S_{M}(T)$ curves from $\mu_{0} H_{0}=0 \mathrm{~T}$ to $\mu_{0} H_{f}=1.5 \mathrm{~T}$ for samples G1 (upper panel), G2 (central panel) and G3 (lower panel). The exponent $n$ is field independent from a value of field $\mu_{0} H \sim 0.25 \mathrm{~T}$ and takes a value close to 1 .

$\Delta S_{M}(T)$ curves present maximum values, as presented in Figure 4 , is another indication that the field dependence of $\Delta S_{M}$ is nearly linear in the temperature span defined by the peaks of the $\Delta S_{M}(T)$ curves. Figure 4(a) shows the temperature dependence of the exponent $n$ for sample G2 (similar behavior for the others sample G1 and G3 has been found). A comparison to the experimental data corresponding to the single-phase sample $\mathrm{Fe}_{77} \mathrm{Co}_{5.5} \mathrm{Ni}_{5.5} \mathrm{Zr}_{7} \mathrm{~B}_{4} \mathrm{Cu}_{1}$ (open symbols) indicates that while the exponent $n$ takes the abovementioned minimum value for single-phase system at the Curie temperature (vertical arrow and dashed line at the Curie temperature $T_{\mathrm{c} \text {, single-phase }}$ ), for multiphase systems the exponent $n$ takes the asymptotic value 1 in the whole ferromagnetic region (horizontal arrow, which is associated with the distribution of the Curie temperatures $\left.\Delta T_{\mathrm{C} \text {, multiphase }}\right)$, getting the asymptotic value 2 in the paramagnetic one. This field dependence represented by the exponent $n$ could be used to design materials with enhanced mag-

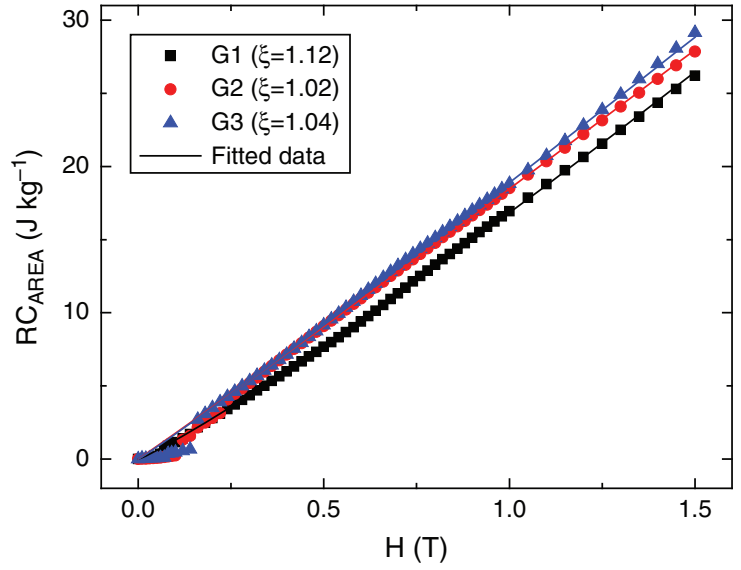

Fig. 5. Field dependence of the refrigerant capacity $R C_{\mathrm{AREA}}$ in the

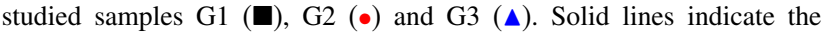
non-linear fit according to Eq. (4) corresponding to experimental data.

netocaloric behavior, showing that magnetic multilayers are a way to increase the magnetic field responsiveness of magnetocaloric materials for moderate magnetic fields.

On the other hand, Figure 4(b) shows that the exponent $n$ takes constant values close to 1 for fields above $\mu_{0} H \sim 0.25 \mathrm{~T}$, which is the field required for magnetic saturation. This behavior of the exponent $n$ is contrary to that presented usually in the literature that indicates a decreasing dependence of exponent $n$ when the magnetic field increases.

The refrigerant capacity $R C_{\mathrm{AREA}}$ of the studied samples has been obtained according to Eq. (2) and its field dependence has been fitted using Eq. (4) in the magnetic field range between $\mu_{0} H=0.25 \mathrm{~T}$ and $\mu_{0} H_{f}=1.5 \mathrm{~T}$, as indicated in Figure 5. The values of the exponent $\xi$ have been indicated in brackets. The results indicate approximately the same dependence on the applied field.

\section{CONCLUSIONS}

Electrodeposition proved to be a flexible technique to produce multilayered materials in which the composition and hence the Curie temperature changes from one layer to another, hence providing an unusual magnetocaloric behavior. The magnetocaloric responses of three $\mathrm{Ni}-\mathrm{Cu}$ based multilayer samples have been investigated. In all the cases studied, the field dependence of $\Delta S_{M}$ is linear in a broad environment of the Curie temperature, demonstrating that nanostructuring is a good strategy to control the way in which magnetocaloric materials respond to the field. Although the studied samples do not present high $\Delta S_{M}$ values, all of them present a large temperature span $\delta T_{\mathrm{FWHM}} \sim 200 \mathrm{~K}$ as a result of the overlapping of the magnetocaloric responses of the existing phases with a broad Curie temperature distribution $\Delta T_{\mathrm{C}} \sim 150 \mathrm{~K}$. In this wide Curie temperature range, the exponent $n$ controlling the 
Magnetic Multilayers as a Way to Increase the Magnetic Field Responsiveness of Magnetocaloric Materials Caballero-Flores et al.

field dependence of the magnetic entropy change exhibits a value close to 1 .

Acknowledgments: The financial support of the Spanish Ministry of Science and Innovation and EU FEDER (Project MAT 2010-20537), the PAI of the Regional Government of Andalucía (Project FQM-6462), the United States Office of Naval Research (Project N00014-11-1-0311) and of the Hungarian Scientific Research Fund (OTKA grants K68612 and NN-79846) is acknowledged. R. Caballero-Flores acknowledges a research fellowship from the Regional Government of Andalucía.

\section{References and Notes}

1. K. A. Gschneidner, Jr, V. K. Pecharsky, and A. O. Tsokol, Rep. Prog. Phys. 68, 1479 (2005).

2. E. Brück, J. Phys. D 38, R381 (2005).

3. A. M. Tishin and Y. I. Spichkin, The Magnetocaloric Effect and Its Applications, 1st edn., Institute of Physics Publishing, Bristol, Philadelphia (2003), p. 475.
4. B. F. Yu, Q. Gao, B. Zhang, X. Z. Meng, and Z. Chen, Int. J. Refrig. 26, 622 (2003).

5. K. A. Gschneidner, Jr and V. K. Pecharsky, Annu. Rev. Mater. Sci. 387, 30 (2000).

6. V. Franco, A. Conde, J. M. Romero-Enrique, and J. S. Blázquez, J. Phys.: Cond. Matter 20, 285207 (2008).

7. V. Franco and A. Conde, Int. J. Refrig. 93, 465 (2010).

8. R. Caballero-Flores, V. Franco, A. Conde, Q. Y. Dong, and H. W. Zhang, J. Magn. Magn. Mater. 322, 804 (2010).

9. T. Mukherjee, S. Sahoo, R. Skomski, D. J. Sellmyer, and Ch. Binek, Phys. Rev. B 79, 144406 (2009).

10. Q. Zhang, S. Thota, F. Guillou, P. Padhan, V. Hardy, A. Wahl, and W. Prellier, J. Phys.: Condens. Matter 23, 052201 (2011).

11. I. Bakonyi, E. Tóth-Kádár, J. Tóth, T. Becsei, T. Tarnóczi, and P. Kamasa, J. Phys. Cond. Matter 11, 963 (1999).

12. E. Pellicer, A. Varea, S. Pané, B. J. Nelson, E. Menéndez, M. Estrader, S. Suriñach, M. D. Baró, J. Nogués, and J. Sort, Adv. Funct. Mater. 20, 983 (2010).

13. C. Bonhote and D. Landolt, Electrochim. Acta 42, 2407 (1997).

14. E. Tóth-Kádár, L. Péter, T. Becsei, J. Tóth, L. Pogány, T. Tarnóczi, P. Kamasa, I. Bakonyi, G. Láng, Á. Cziráki, and W. Schwarzacher, J. Electrochem. Soc. 147, 3311 (2000).

15. W. R. A. Meuleman, S. Roy, L. Péter, and I. Varga, J. Electrochem. Soc. 149, C479 (2002).

16. T. A. Green, A. E. Russell, and S. Roy, J. Electrochem. Soc. 145,875 $\underline{(1998)}$.

Received: 11 May 2011. Accepted: 9 November 2011. 